\title{
Synthesis and Resolution of Chiral Ruthenium Complexes Containing the 1-Me-3-PhCp Ligand
}

\author{
Yue Hu, ${ }^{\mathrm{a}}$ Anthony P. Shaw, ${ }^{\mathrm{b}}$ Hairong Guan, ${ }^{\mathrm{c}}$ Jack R. Norton, ${ }^{*, \mathrm{~d}}$ \\ Wesley Sattler, ${ }^{\mathrm{e}}$ Yi Rong ${ }^{\mathrm{d}}$ \\ ${ }^{a}$ Eastman Chemical Company, Kingsport, Tennessee 37662, United States \\ ${ }^{\mathrm{b}}$ Pyrotechnics Technology and Prototyping Division, U.S. Army RDECOM-ARDEC, Picatinny \\ Arsenal, New Jersey 07806, United States \\ ${ }^{\mathrm{c}}$ Department of Chemistry, University of Cincinnati, Cincinnati, Ohio 45221, United States \\ d Department of Chemistry, Columbia University, New York, New York 10027, United States \\ e Dow Electronic Materials, The Dow Chemical Company, Marlborough, Massachusetts, 01752, \\ United States \\ *e-mail:jrn11@columbia.edu
}

\section{Contents}

Figure S1. Circular Dichroism (CD) spectra of $\left(S_{C p}, S\right)-11$ and $\left(R_{C p}, S\right)-11 . \quad \mathrm{S} 2$

Figure $\mathrm{S} 2 .{ }^{31} \mathrm{P}\left\{{ }^{1} \mathrm{H}\right\}$ NMR spectra of $\left(S_{C p}, S\right)-\mathbf{1 0}\left[\mathrm{BPh}_{4}\right]$ and $\left(R_{C p}, S\right)-\mathbf{1 0}\left[\mathrm{BPh}_{4}\right] \quad \mathrm{S} 3$

Table S1. Summary of Crystallographic Data for $\left(S_{C p}, S\right)-\mathbf{1 1}$ and $\left(R_{C p}, S\right)-\mathbf{1 1} \quad \mathrm{S} 4$ 
Figure S1. Circular Dichroism (CD) spectra of $\left(S_{C p}, S\right)-\mathbf{1 1}$ and $\left(R_{C p}, S\right)$-11. The presence of two chiral centers complicates the $\mathrm{CD}$ signals.

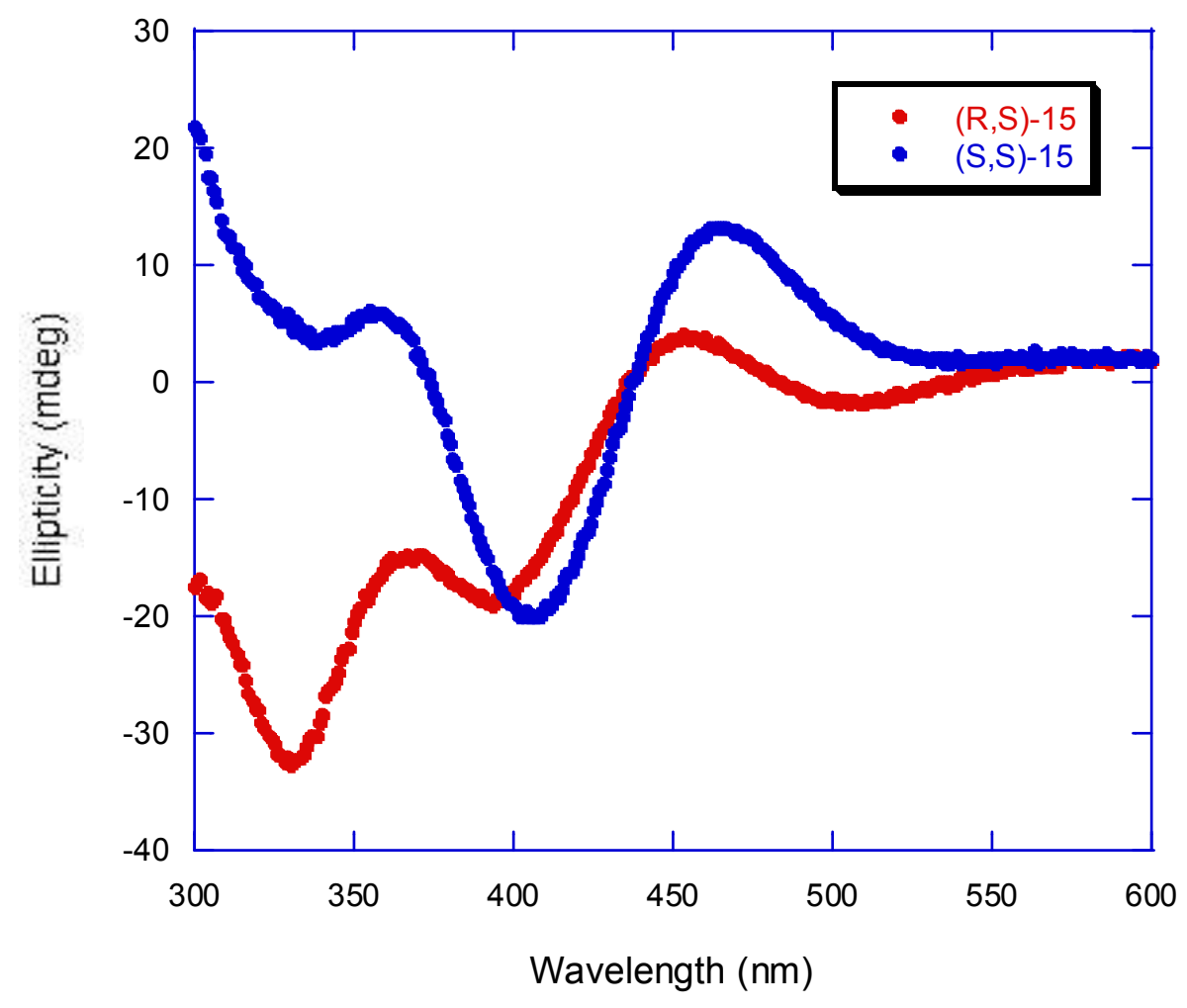


Figure $\mathrm{S} 2 .{ }^{31} \mathrm{P}\left\{{ }^{1} \mathrm{H}\right\}$ NMR spectra of $\left(S_{C p}, S\right)-\mathbf{1 0}\left[\mathrm{BPh}_{4}\right]$ (top) and $\left(R_{C p}, S\right)-\mathbf{1 0}\left[\mathrm{BPh}_{4}\right]$ (bottom) in $\mathrm{CD}_{2} \mathrm{Cl}_{2}$
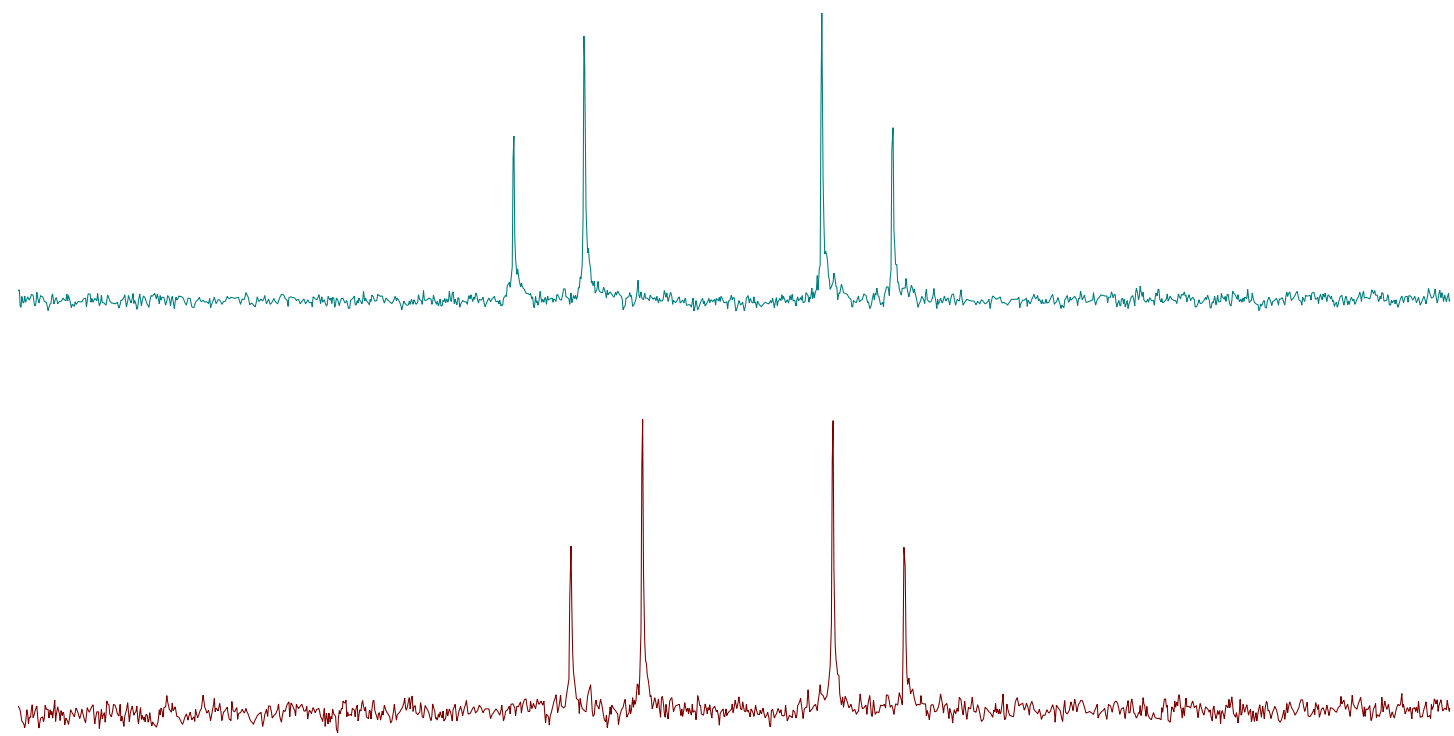

$\begin{array}{llllllllllllllllllllllllllllllllll}14.0 & 13.5 & 13.0 & 12.5 & 12.0 & 11.5 & 11.0 & 10.5 & 10.0 & 9.5 & 9.0 & 8.5 & 8.0 & 7.5 & 7.0 & 6.5 & 6.0 & 5.5 & 5.0 & 4.5 & 4.0 & 3.5 & 3.0 & 2.5 & 2.0 & 1.5 & 1.0 & 0.5\end{array}$ 
Table S1. Summary of Crystallographic Data for $\left(S_{C p}, S\right)-11$ and $\left(R_{C p}, S\right)-\mathbf{1 1}$

\begin{tabular}{ccc}
\hline & $\left(S_{C p}, S\right)-\mathbf{1 1}$ & $\left(R_{C p} S\right)-\mathbf{1 1}$ \\
\hline empirical formula & $\mathrm{C}_{45} \mathrm{H}_{42} \mathrm{P}_{2} \mathrm{RuS}$ & $\mathrm{C}_{45} \mathrm{H}_{42} \mathrm{P}_{2} \mathrm{RuS}$ \\
formula weight & 777.86 & 77.86 \\
temperature $(\mathrm{K})$ & $150(2)$ & $150(2)$ \\
wavelength $(\AA)$ & 0.71073 & 0.71073 \\
crystal system & tetragonal & monoclinic \\
space group & $\mathrm{P} 4(3) 2(1) 2$ & $\mathrm{C} 2$ \\
$\mathrm{a}(\AA)$ & $13.8458(16)$ & $27.049(9)$ \\
$\mathrm{b}(\AA)$ & $13.8458(16)$ & $8.353(3)$ \\
$\mathrm{c}(\AA)$ & $39.210(5)$ & $16.701(5)$ \\
$\alpha(\mathrm{deg})$ & 90 & 90 \\
$\beta(\mathrm{deg})$ & 90 & $98.223(5)$ \\
$\gamma(\mathrm{deg})$ & 90 & 90 \\
volume $\left(\AA^{3}\right)$ & $7516.7(15)$ & $3735(2)$ \\
$\mathrm{z}$ & 8 & 4 \\
$\mathrm{~d}_{\text {calc }}\left(\mathrm{g} / \mathrm{cm}{ }^{3}\right)$ & 1.375 & 1.383 \\
absorption coefficient $\mu$ & 0.589 & 0.593 \\
crystal size $(\mathrm{mm})$ & $0.35 \times 0.20 \times 0.17$ & $0.40 \times 0.10 \times 0.04$ \\
reflections collected & 131182 & 29542 \\
independent reflections & $13464\left(\mathrm{R}_{\text {int }}=0.0525\right)$ & $11247\left(\mathrm{R}_{\text {int }}=0.0784\right)$ \\
data/restraints/param. & $13464 / 0 / 445$ & $11247 / 1 / 445$ \\
$\mathrm{GOF}$ on $\mathrm{F}^{2}$ & 1.236 & 1.002 \\
$\mathrm{R} 1$, wR2 $[\mathrm{I}>2 \alpha(\mathrm{I})]$ & $0.0363,0.0800$ & $0.0340,0.0716$ \\
$\mathrm{R} 1$, wR2 (all data) & $0.0463,0.0836$ & $0.0860,0.0814$ \\
\hline
\end{tabular}

